This article has been accepted for publication in Journal of New Music Research, published by Taylor and Francis. This document is a preprint version of the Authors' Original Manuscript as submitted for anonymous peer review.

The Version of Record of this manuscript has been published and is available in

Journal of New Music Research, 24 ${ }^{\text {th }}$ September 2021, https://www.tandfonline.com/doi/full/10.1080/09298215.2021.1978506 
Motor performance in violin bowing: Effects of attentional focus on acoustical, physiological and physical parameters of a soundproducing action

Emma Allingham ${ }^{\mathrm{a} 1^{*}}$, Birgitta Burger $^{\mathrm{a} 2}$ and Clemens Wöllner ${ }^{\mathrm{a} 3}$

${ }^{a}$ Institute of Systematic Musicology, University of Hamburg, Hamburg, Germany;

*emma.allingham@uni-hamburg.de, ${ }^{1}$ ORCiD: https://orcid.org/0000-0001-5270-4059

${ }^{2}$ ORCiD: http://orcid.org/0000-0002-0694-3582

${ }^{3}$ ORCiD: http://orcid.org/0000-0002-8508-3508

Universität Hamburg, Institute of Systematic Musicology, ERC-Project SloMo, Alterterrasse 1, 20354 Hamburg, Germany 


\title{
Motor performance in violin bowing: Effects of attentional focus on acoustical, physiological and physical parameters of a sound- producing action
}

\author{
Violin bowing is a specialised sound-producing action, which may be affected by \\ psychological performance techniques. In sport, attentional focus impacts motor \\ performance, but limited evidence for this exists in music. We investigated \\ effects of attentional focus on acoustical, physiological, and physical parameters \\ of violin bowing in experienced and novice violinists. Attentional focus \\ significantly affected spectral centroid, bow contact point consistency, shoulder \\ muscle activity, and novices' violin sway. Performance was most improved when \\ focusing on tactile sensations through the bow (somatic focus), compared to \\ sound (external focus) or arm movement (internal focus). Implications for motor \\ performance theory and pedagogy are discussed.
}

Keywords: focus of attention, violin performance, sound-producing action, motor skills, motion capture

\section{Introduction}

The rich, expressive sound of the violin has been described as imitating the quality of the human voice (Deutsch, 2011). Learning to produce this sound requires highly specialised fine motor skills, developed over years of practice (Konczak, Vander Velden, \& Jaeger, 2009), and while mathematical understanding of bowed string motion is well documented (e.g. Schoonderwaldt, 2009a), little is known about how psychological performance techniques affect these skills. A human's motor control of a musical instrument exists within a cognitive system involving thoughts and mental processing (Desmet et al., 2012), meaning that a musician's psychological approach to performance may explicitly or implicitly influence their physical manipulation of sound. In sport research, a wealth of studies have found that performance of a motor action can be improved by focusing attention on the environmental effects of an action compared 
to focusing on internal movement processes (for a review see Wulf, 2013), but little is known about these effects in instrumental music making. Violin playing offers a particularly interesting context to explore this topic due to the sophisticated psychomotor skills required for sound manipulation. The current study explores effects of the psychological performance strategy 'focus of attention' (FOA) on the system of sound production in violin playing.

\section{Sound production in violin playing}

Sound production on the violin is controlled via three main parameters: bow speed, bow force (downward pressure on the string), and the distance between the bow's position of contact with the string and the violin bridge (i.e. bow contact point, Perez-Carrillo, 2016; Schoonderwaldt, 2009b). Within this dynamic system of sound creation, violinists manipulate these parameters to achieve artistic expression, while simultaneously maintaining a delicate balance between parameters to maintain tone quality. Thus, subtle alterations to bowing variables can affect the sound produced. Indeed, the mathematical study of how string instruments produce sound has received considerable attention. For example, it has been shown that the spectral centroid of a violin tone is mainly controlled through bow force (Schoonderwaldt, 2009b), while volume of playing tends to be controlled through bow contact point, and skewness of the bow causes increased noise in the audio signal (Edgerton, Hashim, \& Auhagen, 2014). Yet, the question of how psychological approaches to performance may affect motor behaviours within this system of sound production remains largely unexplored. In describing the process of sound-producing actions, Jensenius (2007) depicted an actionsound chain of cognition in which neurological activity in the brain leads to physiological muscle activity, physical movement of limbs, mechanical control of the instrument and eventually acoustical impacts on the environment (Figure 1). In the 
current study, we suggest that an additional psychological element may influence sound production. Thus, we utilise the action-sound chain framework to explore effects of a psychological performance technique on physiological, physical and acoustical aspects of violin tone production.

** Figure 1 about here**

Measuring acoustic changes in violin tone production can be a complex endeavour. Research on musical timbre has mapped verbal descriptions of sound to multidimensional spaces (e.g. Alluri \& Toiviainen, 2009) showing that this aspect of sound is complex and multifaceted. In terms of violin tone, there does not seem to be a clear understanding of the acoustic features that constitute a "good" tone quality, and indeed tone quality is ultimately a subjective issue. Nonetheless, acoustic features of string sound can provide information about the means of sound production. For example, machine learning models have been successfully applied to the prediction of violin bowing parameters (Perez-Carrillo, 2019), and assessing violin tone quality (Giraldo, Ramirez, Waddell, \& Williamon, 2017; Giraldo et al., 2019) from acoustic features of the sound. Acoustic analysis has also been used to characterise the sound of different violins (Nagyvary, 2013), and to predict cello performer identity (Chudy, 2016). Thus, low level acoustic features might be applied to violin sound to describe changes in tone production. For example, the root mean square (RMS) of an audio signal provides information about the loudness of the sound (Hove, Vuust, \& Stupacher, 2019), roughness gives a measure of sensory dissonance (Eerola, Ferrer, \& Alluri, 2012), and spectral centroid can be considered a measure of the brightness of tone (Edgerton et al., 2014; Schoonderwaldt, 2009b). 


\section{Focus of attention in motor skill performance}

In order to explore how a change in psychological approach might affect this system of sound production, we turn to the field of sports performance psychology. Research on the topic of focus of attention (FOA) in sport has shown that the object of a performer's thoughts can affect their motor performance. In this paradigm, performers are given verbal instructions on whether to focus their attention on the movements they make to perform a task (internal focus) or on the environmental effect or wider goal of the task (external focus, Wulf \& Lewthwaite, 2016). Results widely show that an external focus produces superior performance for many types of gross motor skill (e.g. Neumann, 2019; Wulf, 2013). This phenomenon is explained by the constrained action hypothesis (CAH, McNevin et al., 2002; Wulf, McNevin, \& Shea, 2001) which states that attention to the movements of an automatised skill brings implicit processes into conscious awareness, disrupting automaticity and leading to impaired performance. This explanation is based on the idea of common coding theory (Wulf, Höß, \& Prinz, 1998), that actions are more efficient when planned in anticipation of their sensory outcome. In support of the mechanisms behind constrained action, evidence for the explicit monitoring theory of choking under pressure likewise suggests that failure of a motor skill in high-pressure situations is caused by attempts to control proceduralised skills (Beilock \& Carr, 2001). Some studies have also shown a distance effect such that external foci more distal from the body (e.g. on a point in the distance) produce superior performance compared to external focuses more proximal to the body (e.g. on a piece of equipment, Alishah, Ates, \& Ahmadi, 2017; Bell \& Hardy, 2009; McKay \& Wulf, 2012; McNevin, Shea, \& Wulf, 2002; Porter, Anton, \& Wu, 2012; Singh \& Wulf, 2020). Furthermore, an internal focus can affect measurable motor behaviours such as less efficient muscle use (Vance, Wulf, Töllner, McNevin, \& Mercer, 2004), and 
freezing of the degrees of freedom of joint motion (Wulf \& Dufek, 2009). These findings exemplify how a seemingly small change in psychological approach can impact both performance of a specific motor task, and measurable changes to the whole motor control system.

\section{Attentional focus in music}

A few previous studies have laid important groundwork for understanding FOA effects in music making. Duke, Cash, and Allen (2011) found an effect of attentional focus on skill transfer in a piano task, such that temporal evenness of playing two alternating notes was improved by focusing on either sound, or the piano hammers (distal external foci), compared to the piano keys, (proximal external focus) or the fingers (internal focus). These results were seminal in supporting the $\mathrm{CAH}$ for a musical task involving auditory feedback. Similarly, Atkins (2017) found that trained singers received higher expert ratings when performing under distal external foci compared to internal and proximal external foci, while Mornell and Wulf (2019) found that an external focus on musical expression compared to an internal focus on technical accuracy improved expert ratings of both musicality and technical accuracy for various kinds of expert instrumentalists.

On the other hand, some music studies have failed to replicate the FOA effects found in sport. Atkins (2013) found a main effect of FOA on expert ratings of untrained singers' performances, but differences between the conditions were unclear. Contrary to the $\mathrm{CAH}$, performances were most often ranked as best under an internal focus on feeling vibrations in the zygomatic arch (cheekbones), compared to feeling vibrations in the throat, focusing on a microphone, or focusing on a distal point on the wall. In this study, the internal focus instructions were centred around tactile sensory feedback rather than movement in itself, and this quality of the internal foci may have affected results. 
Indeed, the zygomatic arch focus might actually be considered an external focus, as it diverted attention away from the main source of motor activity (the larynx), and focused not on movement, but on sensation. Similarly, two studies on woodwind playing also failed to support the $\mathrm{CAH}$, with no significant effects of focus condition on performance outcomes (Stambaugh, 2017, 2019). The author likewise suggests that as tactile feedback plays an important role in controlling sound production in woodwind playing, any attention to tactile sensations brought about as a consequence of the internal focus instructions might have interfered with constrained action effects. It is also noted that basic sound production using woodwind instruments is more complex (i.e. involves coordination of both hands and breathing) than previously tested tasks of piano playing and singing, which also may have affected results (Stambaugh, 2017). Although Mornell and Wulf (2019) found support for the CAH in a variety of instrumental performances, their conception of the external focus as "on musical expression" and the internal focus as "on technical accuracy" is not directly comparable with the other studies discussed here. Therefore, the current literature on FOA in music making is lacking in evidence for the $\mathrm{CAH}$ in complex instrumental sound production. Furthermore, the potential influence of bringing attention to tactile feedback in instrumental playing warrants further exploration, as does the study of measurable motor outcomes much as muscle activity and motion features.

\section{Tactile sensory awareness in music performance}

In support of indications from previous FOA research in music that attention to tactile sensory feedback may be beneficial to performance, other areas of research highlight the role of attention to body sensations. For example, somatic training methods such as the Alexander technique (AT), Feldenkrais method, and body mapping are widely thought to improve performance and reduce risk of injury through the cultivation of 
sensitivity to body sensations, muscle tension and movement habits (Davies, 2020; Lee, 2018; Slade, Comeau, \& Russell, 2020). While it has been argued that this somatic approach contradicts $\mathrm{CAH}$ theory because it encourages attention to the body, and to process rather than outcome (Ives, 2003; Shusterman, 2009), others have argued that the somatic approach in fact encourages external FOA, by focusing on quality of movement, rather than movement itself (Mattes, 2016). In support of the somatic approach, studies have found that expert performers under pressure tend to focus on physical sensations such as breathing or posture (Buma, Bakker, \& Oudejans, 2015; Kokotsaki \& Davidson, 2003), and that attention to body sensations may play a role in preventing overuse injuries (Batson, 2007). Researchers have highlighted the importance to learning of haptic feedback for woodwind and brass players (Dries, Vincken, Loeckx, Schuermans, \& Dirckx, 2017; Stambaugh, 2017, 2019), while string playing pedagogy also indicates the value of developing kinaesthetic sensing in learning to control sound via the bow (Cotik, 2019). Therefore, attention to tactile feedback may influence production of sound in string playing. To our knowledge, no previous study of FOA in music performance has investigated how focusing on tactile sensory feedback through an instrument might compare with internal and more distal external FOA.

\section{Expertise and FOA}

FOA research in sport has shown that the $\mathrm{CAH}$ applies to performers of various levels of expertise (Wulf, 2013). However, in a recent study it was shown that beginner volleyball players may benefit more from a proximal external focus, and experts from a distal one (Singh \& Wulf, 2020). The authors suggest that their proximal external focus which utilised imagery about arm angle without referencing arm movement per se, avoided constrained action by diverting attention away from motor mechanics, while also bringing awareness to action-execution technique. While experts were able to 
achieve their best performance by focusing distally on a target, beginners benefitted from the extra attention to technical detail allowed by the proximal external focus. This finding might also be considered in light of implicit motor learning theory (Chambaron, Berberian, Delbecque, Ginhac, \& Cleeremans, 2009). For example, it has been suggested that reported benefits of motor learning without explicit instructions (i.e. implicit learning) might be underpinned by constrained action effects, in that explicit movement instructions might promote an internal focus, while implicit learning conditions encourage an external focus (Shea, Wulf, Whitacre, \& Park, 2001). The findings that beginners benefit from a technique-centred external focus might offer a way in which implicit learning can be achieved while still concentrating on technique. In musical tasks, interactions of FOA with expertise remain unclear. Atkins (2017) found beneficial effects of an external focus for trained singers, while Atkins (2013) found no clear benefits of external focus for untrained singers. In contrast, Duke, Cash, and Allen (2013) found that less experienced pianists benefitted from a more external FOA, while expert pianists were unaffected. Violin bowing is a particularly complex motor skill, which has been characterised as learning to coordinate five potential degrees of freedom of arm motion into a two-degrees-of-freedom bowing action, and which beginners must accumulate at least 700 hours of practice to achieve (Konczak et al., 2009). Thus, violin bowing is a particularly interesting context for exploring effects of FOA and expertise.

\section{The whole body in violin playing}

It is important to consider that a violinist's mechanisms of bow control are situated within the musician's whole body. Whole body movement contributes to perceptions of expressive music performance (e.g. Dahl \& Friberg, 2007), and in violin pedagogy it is considered important that the whole body is able to move freely so that stiffness and 
excess muscle tension are avoided (Medoff, 1999; Roos, 2001). Furthermore, it has been shown that for singers, instructions to minimize body movement cause decreased sound pressure levels (Turner \& Kenny, 2011), and in cello playing, head and torso movements contribute to the player's ability to generate fluid bowing and good quality sound (Rozé, Aramaki, Kronland-Martinet, \& Ystad, 2020). These findings highlight how overall freedom of body motion might impact sound quality. Thus, a measure of whole-body motion such as instrument sway may be considered a more global aspect of the physical stage of a sound-producing action. Furthermore, more static postures have been found to be associated with increased pain, while increases in micro movements are associated with less pain (Vergara \& Page, 2002), indicating that postures which are "too still" may negatively impact the body. In addition, the physiological stage of sound production can be investigated using electromyography (EMG). EMG muscle activity can provide information about energy expended through muscle use - another important aspect of the motor control system. Excess muscle tension is a common health issue among instrumental musicians (Burkholder \& Brandfonbrener, 2004), and something that somatic training methods aim to reduce. FOA studies in sport have shown that EMG activity is decreased under an external focus compared to internal (e.g. Marchant \& Greig, 2017; Neumann \& Brown, 2013; Vance, Wulf, Töllner, McNevin, \& Mercer, 2004), but this has not before been investigated in an instrumental music context. Therefore, exploring how FOA may affect global motor behaviour and muscle activity will provide a wider picture of how psychological performance strategies can affect different aspects of a sound production task.

In summary, sound production in violin playing is relatively well understood in terms of the mathematical relationships between bowing parameters and string motion, but little research exists on how psychological performance techniques may influence 
bowing action. A useful cognitive framework, the sound-producing action chain (Jensenius, 2007) depicts the various stages of sound production from neurological to acoustical, and we suggest that the additional psychological element should be explored. In sports research, such a psychological effect on motor performance has been observed in research on attentional focus. That is, motor performance is improved by adopting an external focus on task goals compared to an internal focus on movement processes (Wulf, 2013), a phenomenon explained by the Constrained Action Hypothesis (McNevin et al., 2002; Wulf, McNevin, \& Shea, 2001) . Further, this constrained action effect has been shown to influence other aspects of the motor system such as motion features, and muscle activity (Vance et al., 2004; Wulf \& Dufek, 2009). However, evidence for these effects in music performance is limited. In addition, some evidence suggests that attention to tactile sensory feedback could be beneficial in instrumental music making (Davies, 2020; Lee, 2018; Slade et al., 2020; Stambaugh, 2017), which may have implications for finding an optimal focus of attention for music performance.

\section{The Current Study}

\section{Aims}

The current study aimed to investigate how a psychological performance approach might affect motor skill performance during violin tone production. To this end, we applied the FOA paradigm founded in sports psychology to a simple violin sound production task, for both experts and novices. To gain a detailed view of effects on the motor system we examined outcomes at various stages of the sound-producing action chain (see Figure 1), namely physiological (EMG muscle activity), physical (technical bowing and scroll sway motion parameters) and acoustical (MIR features of the sound produced). While scroll sway may not be a direct physical aspect of sound production, 
it is considered here as part of the sound-producing action chain as whole-body motion may influence production and perceptions of sound (see Introduction).

We aimed to compare effects of internal and external foci with a novel "somatic" focus on tactile sensory feedback through the bow, which also served as a proximal external focus, allowing testing of the distance effect. The internal instruction aimed to bring attention to the internal mechanics of the task (arm movements), and the distal external instruction aimed to bring attention to the external goal of sound production, while the somatic focus aimed to direct attention towards tactile sensations resulting from the action, through reference to the musical instrument. Thus, the somatic focus can be considered a proximal external focus, as it does not refer to movement mechanisms and is situated closer to the body than the sound focus. Additionally, the somatic focus was intended to provide an external focus which is still grounded in bodily awareness; i.e. the focus is reliant on attending to body sensations rather than ignoring the body completely. In this way, the somatic focus was intended to mirror the kind of awareness which may occur as part of somatic training methods. Therefore, the somatic focus provided a middle ground between the hypothesised benefits of an external focus, and the theoretical implications that a grounding in sensory bodily awareness may improve performance.

We hypothesised that:

1) Focus instructions would affect motor control of sound production at physiological, physical, and acoustical stages. In accordance with the $\mathrm{CAH}$, we predicted that distal external and proximal external (somatic) foci would benefit motor performance relative to the internal focus. As a somatic focus has not been tested before in this context, we did not predict differences between somatic and distal external. 
2) There would be differences in performance outcomes and attentional focus effects between novices and experts. As expertise effects on constrained action are not well established in music tasks, we did not predict the direction of this effect.

The physiological, physical and acoustical stages of sound production were measured through EMG muscle activity, motion capture of bowing parameters and violin sway, and music information retrieval, respectively.

\section{Materials and Methods}

\section{Participants}

Thirty-three right-handed participants ( 18 female, mean age $=24.97$ years, $S D=4.80)$ were recruited. One participant was excluded from the analysis as their level of training was not enough to be considered experienced, but too much to be considered a novice. This resulted in a sample of 32 participants ( 18 female, mean age $=24.94, S D=4.87$ ), all of whom were compensated $€ 10$ for taking part. All participants played a musical instrument (mean years played $=15.20, S D=6.35) .16$ participants comprised the novice group, having never played a string instrument before, and 16 qualified as experienced violin or viola players, having at least 7 years of training in their instrument.

\section{Equipment and experimental set-up}

The experiment was carried out in a $5 \mathrm{~m} \times 5 \mathrm{~m}$ room outfitted with eight infrared-based motion capture cameras (Qualisys Oqus). The violin used was a Fastoso intermediate model which was mounted with an AKG Harman C411PP contact microphone. Red stickers were placed on the stick of the bow to mark the middle section of the bow. The 
sound was recorded via Audio Desk software and a MOTU 828MK3 audio interface, while motion capture data was recorded via Qualisys Track Manager (QTM) software. Audio and motion capture were synchronised via SMPTE timecode. Participants were outfitted with motion capture jackets and caps, and 24 reflective markers were placed on the participant's body as shown by grey markers in Figure 2 (right panel). For the lower body, markers were attached directly to the participant's clothes. In addition, five markers were placed on the violin and bow, as shown by the black markers in Figure 2 (right panel). Trigno Delsys wireless surface EMG sensors recorded muscle activity using QTM software in synchrony with the motion capture. The wireless EMG sensors were placed on the participant's bicep, tricep, and deltoid muscles of the right arm (see Figure 2, left panel).

** Figure 2 about here**

\section{Procedure}

Before the start of the experiment, participants gave written informed consent in accordance with the Local Ethics Committee guidelines, and filled out a brief demographic questionnaire including their musical training history. Next, EMG sensors were positioned over the belly of the muscle, parallel to muscle fibres, in accordance with SENIAM guidelines (Surface ElectroMyoGraphy for the Non-Invasive Assessment of Muscles, see www.seniam.org). The signal to noise ratio was then visually checked for each muscle using the SENIAM recommended movements. Next, participants were outfitted with the motion capture jacket, cap, and markers, and there was a short training session in which novices were taught the basics of holding the violin and bow and how to carry out the experimental tasks (see below), while experienced violinists were simply taught the experimental tasks. For novices, this session lasted approximately 15 - 20 minutes, and for experienced violinists, 
approximately 10 minutes. Participants were instructed to keep their visual gaze on the violin A-string during the task.

\section{Task}

Participants performed 4 bows (starting on a down-bow) on the open A-string (tuned to A4) in response to a metronome, set to $30 \mathrm{bpm}(\mathrm{IOI}=2000$ milliseconds $(\mathrm{ms})$ ). Participants were instructed that the goals of the task were: 1) to play in time with the metronome, 2) to use the middle section of the bow as defined by the stickers, and 3) to play with a good sound. A good sound was defined as: 1) consistent volume and tone quality, 2) avoiding scratching, scraping or squeaking sounds, 3) smooth bow changes. They were instructed to create a resonant tone at a medium mezzo-forte dynamic.

In an initial practice round, participants carried out the task with no focus instruction. The task was then performed under three focus conditions, counterbalanced in order across participants: internal focus, external focus, and somatic focus. For each condition, participants performed three trials of the task. Before each new focus condition, participants sat quietly for one minute to minimise carry-over effects between conditions. Focus instructions were given verbally, and reinforced for each repetition of the task. After each focus condition, participants were asked to verbally report what they had been thinking about as a check to see if they had followed the focus instructions. After the experiment was complete, participants were debriefed about the purpose of the experiment. The focus instructions were as follows:

- Internal: Focus your attention on the movement in your right arm.

- External (distal external): Focus your attention on the sound you produce.

- Somatic (proximal external): Focus your attention on the resistance of the bow against the string. 


\section{Data Analysis}

\section{Ability to follow the focus instruction}

To gain an overview of how well participants followed the focus instructions we carried out a qualitative analysis of their reported thoughts after each condition. Participants' answers were transcribed by the experimenter and later were qualitatively coded as having clearly followed the instruction (1) or not (0). Comments were coded with a 1 if the participant reported: 1) that they were thinking about the object of the focus instruction or that they were thinking about "the focus", or 2) if they directly reported being able to do the focus, enjoying the focus or trying to do the focus. Comments were coded with a 0 if participants 1 ) directly reported difficulty with the focus instruction or 2) if their reported thoughts were completely irrelevant to the focus, implying distraction. $93 \%$ of the overall comments were coded with a 1 , indicating a high rate of success. No participant received a score of 0 for all three conditions. We chose not to exclude any participants on the basis of these comments, as even though they might have exhibited difficulties with following the focus instructions, they may have still been affected by the instructions at an implicit level.

\section{Control measures}

With the bowing task instructions, we aimed to control for tempo and speed of bow used, however small deviations in these parameters were possible. Therefore, to check that these variables were adequately controlled across focus conditions, we ran mixed ANOVAs on the outcome variables 'length of task' (i.e. mean time taken to complete a trial), and bow velocity. The within-subjects factor was condition, and the betweensubjects factor was expertise. Results of main condition effects are displayed in Table 1. No further expertise or interaction effects were found. 
** Table 1 about here **

As there was no significant difference in these variables between focus conditions, we deemed it unnecessary to control further for length of task or speed of bow in the rest of the analyses. Regarding small deviations in length of recordings, many of the outcome variables are expressed as mean or standard deviation values over time, meaning that small variations in length should not affect results.

\section{Audio}

Audio files were first segmented using SMPTE code to match the time series of the motion capture recordings. Audio was then processed using the MIR toolbox (Lartillot \& Toiviainen, 2007) and custom code in MATLAB. Each audio clip was trimmed from the start to the end of the audio waveform to exclude silence. All trials were visually inspected for signal quality, and 16 trials (4.6\% of total trials) were excluded from the analysis due to pops/cracks in the audio signal. If more than 2 trials per condition were deemed poor quality, the participant was excluded completely from the analysis. Two participants were fully excluded on this basis, and an additional participant was excluded because the original audio files were lost. This resulted in a total of 29 participants for the audio analysis.

Segmentation of the audio signal

In order to assess tone quality on the steady part of the sound without the bow changes, we segmented the audio into "bow" and "bow change" sections. The time points of bow changes were detected using the MIRpeaks function, with visual inspection and parameter adjustments for each recording. The "bow change" sections comprised a window around the bow change ( $20 \%$ of the length of the previous bow), and "bow" 
sections consisted of the rest of the signal. This size of the window was chosen based on a visual inspection of the data and was deemed to be an appropriate window size. A segmentation example is displayed in Figure 3. MIR features were then applied only to the "bow" sections, to give an indication of the quality of the sound regardless of the bow changes.

** Figure 3 about here**

\section{MIR features}

Based on previous literature, we selected an initial set of MIR features to measure acoustic qualities of the sound produced during the task (see Table 2). While these three measures are not exhaustive of the possible changes to tone quality which could occur, they were considered to be the most relevant acoustic features to violin sound production technique. For details of feature derivation see the MIR Toolbox manual (Lartillot \& Toiviainen, 2007). Each feature was derived using a windowed analysis (window length $=25 \mathrm{~ms}$, overlap $=0.5$ ), from which the mean and standard deviation over time were calculated.

**Table 2 about here**

\section{Motion Capture}

In QTM software, data were labelled and trimmed via a visual inspection from the start to the end of the bowing action. Finishing gestures at the end of bowing were excluded, as the aim was to examine the kinematics of technical bow movements during sound production. Gaps were filled in QTM using linear interpolation with all gaps visually inspected, so that at least $95 \%$ of the trajectory was filled. Any further small gaps were filled later in MATLAB, also using linear interpolation. For the violin, as it is a rigid 
structure, QTM's relational gap-fill method was utilised, employing linear interpolation within a local coordinate system defined by the available violin markers. For the current study, only the bow and violin markers were analysed (see black markers in Figure 2). The violin scroll marker was used to indicate instrument sway, while technical aspects of sound production were derived from the bow markers. Data were then processed in MATLAB using the Motion Capture Toolbox (Burger \& Toiviainen, 2013). For analysis of the bow, data were converted to a local co-ordinate system in which data were expressed in relation to the violin with the $\mathrm{X}$-axis parallel to the violin bridge (see Figure 4), and the origin positioned at the lower left corner of the violin. This controlled for movement of the violin and individual height differences. For analysis of bow motion, two secondary markers were created by averaging the two bow markers to create a mid-bow marker, and averaging the two markers at the lower bout of the violin to create a "mid-base" marker, which served as a reference for the bow contact point measure (see below). One participant was excluded from the scroll sway analysis due to a completely missing scroll marker in their motion data. From the bow measures, two trials (from two separate participants, $0.6 \%$ excluded trials) were excluded due to poor quality recordings (i.e. missing trajectories).

** Figure 4 about here **

Bow contact point

The contact point of the bow refers to the bow's positioning on the string relative to the bridge. We calculated mean contact point as the difference between the mean position of the mid-bow in the Y-dimension (i.e. perpendicular to the violin bridge), and the mean position of the mid-base in the Y-dimension, in millimetres ( $\mathrm{mm})$. Smaller values represent playing closer to the bridge, and bigger values further from the bridge. 
Consistency of contact point was calculated as the standard deviation of the position of the mid-bow in the Y-dimension.

Scroll sway (freedom of motion)

We calculated scroll sway in the medio-lateral axis in millimetres $(\mathrm{mm})$, as a measure of freedom of overall body motion. Scroll sway was operationalised as the standard deviation of the scroll marker position data in the $\mathrm{Y}$ axis. This approach to deriving sway has been used successfully in previous research on body sway (i.e Riley, Stoffregen, Grocki, \& Turvey, 1999). Scroll sway was derived from the global coordinate system to represent a wider range of motion including both upper body and violin motion. As the measure used was standard deviation of position data, individual differences in the angle of the violin to the body should not influence the measure. Similarly, as only Y axis information was used, differences in participant height or arm length would also not affect the measure.

Bow acceleration

Acceleration of the bow was derived from the three-dimensional position data, in millimetres per second squared $\left(\mathrm{mm} / \mathrm{s}^{2}\right)$ and then the Euclidean norm was derived to provide one value across three dimensions. These values were then averaged over time.

\section{Electromyography}

EMG data were band-pass filtered at $20-450 \mathrm{~Hz}$ within the wireless sensor, and further processed using custom software in MATLAB. All data were visually inspected, and 4 trials containing large artefacts were excluded from the analysis $(0.3 \%$ total excluded trials), although no participants had more than one trial per condition excluded. One 
participant was excluded from the trapezius muscle analysis due to a loose sensor. Data were mean centred and full-wave rectified. A moving RMS filter was applied with a window of $50 \mathrm{~ms}$ and overlap of $25 \mathrm{~ms}$, and data was normalised (max-min) to control for individual differences. The mean RMS value was then calculated in millivolts (mV), to represent the power of muscle activity during each trial, and values were averaged across trials.

\section{Statistical Analysis}

All variables were screened for outliers such that values more or less than three standard deviations from the mean were excluded. Total outlier exclusions were $0.02 \%$ of MIR data, $0.01 \%$ motion capture data, and $0.01 \%$ EMG data. For each dependent variable, a mixed ANOVA was carried out with within-subjects factor 'Focus Condition' (3) and between-subjects factor 'Expertise' (2). Alpha threshold of statistical significance was set at .05 . Where Mauchley's test of sphericity was violated, the Greenhouse-Geisser corrected degrees of freedom are reported. To follow up significant effects of condition, pairwise comparisons were conducted with Bonferroni corrections, and to follow up interaction effects, separate ANOVAs were carried out for each expertise group.

\section{Results}

\section{Acoustic features}

For mean spectral centroid, a main effect of Focus Condition was found $(F(1.52,38)=$ $\left.3.65, p=.047, \eta^{2}=.13\right)$. with Bonferroni-corrected pairwise comparisons revealing that the somatic condition $(M=1439.30 \mathrm{~Hz}, S E=55.07 \mathrm{~Hz})$ resulted in significantly higher spectral centroid compared to internal $(M=1327.16 \mathrm{~Hz}, S E=24.91 \mathrm{~Hz} ; p=$ $.045)$, with no significant differences to external $(M=1341.97 \mathrm{~Hz}, S E=34.69 \mathrm{~Hz})$. This 
result implies a brighter tone quality in the somatic condition (Figure 5).

** Figure 5 about here**

No main effects of Focus Condition were found for $S D$ spectral centroid $\left(F(2,52)=1.60, p=.212, \eta^{2} p=.06\right)$, mean $\operatorname{RMS}\left(F(1.54,41.49)=0.25, p=.720, \eta^{2} p=\right.$ $.009), S D$ RMS $\left(F(1.38,40.12)=0.95, p=.366, \eta^{2} p=.03\right)$, mean roughness $\left(F(1.63,40.83)=0.19, p=.782, \eta_{p}^{2}=.008\right)$ or $S D$ roughness $(F(1.43,35.85)=0.44, p=$ $\left..580, \eta^{2} p=.02\right)$. No significant interactions were found. However, significant Expertise effects were found for all MIR variables apart from mean spectral centroid (Table 3), indicating that experienced violinists played quieter and with less roughness than novices, and had higher consistency than novices in all acoustic features.

** Table 3 about here**

\section{Motion Capture}

\section{Bow contact point}

For mean contact point there was no effect of Focus Condition $(F(2,56)=1.29, p=$ $\left..284, \eta^{2} p=.04\right)$, and no interaction effect. There was an effect of Expertise $(F(1,28)=$ $\left.24.84, p<.001, \eta^{2}{ }_{p}=.47\right)$, such that experienced violinists $(M=141.15 \mathrm{~mm}, S E=$ $1.97 \mathrm{~mm})$ had a contact point further from the bridge compared to novices ( $M=$ 127.72mm, $S E=1.84 \mathrm{~mm})$.

For $S D$ contact point, there was a main effect of Focus Condition $(F(1.60,48.12)$ $\left.=4.98, p=.016, \eta_{p}^{2}=.14\right)$, and no interaction effect. There was no effect of Expertise $\left(F(1,30)=0.71, p=.406, \eta^{2} p=.02\right)$. For the effect of Focus Condition, pairwise comparisons showed a significant difference between external and somatic $(p=.042)$, such that standard deviation of contact point was lower in somatic $(M=6.042 \mathrm{~mm}, S E=$ $0.35 \mathrm{~mm})$ than external $(M=7.07 \mathrm{~mm}, S E=0.48 \mathrm{~mm})$. These results indicate that 
consistency of bow-string contact point improved in the somatic condition compared to the external condition. There were no significant differences compared to internal $(M=$ $6.17 \mathrm{~mm}, S E=0.40 \mathrm{~mm}$, Figure 6).

**Figure 6 about here **

\section{Scroll sway}

For scroll sway, there was no main effect of Focus Condition $(F(2,54)=1.33, p=.273$, $\left.\eta^{2} p=.05\right)$, but there was a significant effect of Expertise $\left(F(1,27)=11.04, p=.003, \eta^{2} p\right.$ $=.29)$, and an interaction effect $\left(F(2,54)=3.93, p=.025, \eta^{2} p=.13\right)$. Experienced violinists $(M=10.86 \mathrm{~mm}, S E=0.91 \mathrm{~mm})$ displayed more scroll sway than novices $(M=$ $6.78 \mathrm{~mm}, S E=0.82 \mathrm{~mm})$. Following up the significant interaction effect, separate ANOVAs showed a significant effect of Focus Condition for novices $(F(2,30)=6.33, p$ $\left.=.005, \eta_{p}^{2}=.30\right)$, and no effect for experienced violinists $\left(F(2,24)=1.70, p=.204, \eta_{p}^{2}=\right.$ .12). Pairwise comparisons for the novice group revealed significantly more scroll sway in the somatic condition $(M=7.92 \mathrm{~mm}, S E=0.75 \mathrm{~mm}, p=.003)$ compared to internal $(M=6.26 \mathrm{~mm}, S E=0.64 \mathrm{~mm})$. There was also a marginally significant difference between somatic and external conditions $(M=6.16 \mathrm{~mm}, S E=0.82 \mathrm{~mm}, p=.050$, Figure 7).

** Figure 7 about here $* *$

\section{Bow Acceleration}

There was no main effect of Focus Condition on mean acceleration $(F(1.47,41.16)=$ $\left.2.12, p=.144, \eta^{2} p=.07\right)$, and no further interaction or Expertise effects. For $S D$ acceleration, there was no main effect of Focus Condition $(F(1.08,29.18)=1.51, p=$ $\left..231, \eta^{2}=.05\right)$. There was a significant effect of Expertise $(F(1,27)=6.24, p=.019$, 
$\left.\eta_{p}^{2}=.19\right)$, such that experienced violinists $\left(M=712.54 \mathrm{~mm} / \mathrm{s}^{2}, S E=67.35 \mathrm{~mm} / \mathrm{s}^{2}\right) \mathrm{had}$ more variable bow acceleration than novices $\left(M=470.32 \mathrm{~mm} / \mathrm{s}^{2}, S E=69.72 \mathrm{~mm} / \mathrm{s}^{2}\right)$, and there was no further interaction effect.

\section{$E M G$}

A main effect of Focus Condition was found for the deltoid $(F(1.35,40.50)=6.34, p=$ $\left..010, \eta_{p}^{2}=.17\right)$ and the tricep $\left(F(2,58)=4.01, p=.023, \eta^{2} p=.12\right)$ muscles, with no Expertise effects or interactions (Figure 8). For the deltoid, a Bonferroni corrected pairwise comparisons showed significant difference between internal $(M=36.46 \mathrm{mV}$, $S E=4.33 \mathrm{mV})$ and somatic $(M=33.16 \mathrm{mV}, S E=3.89 \mathrm{mV}, p=.023)$, while the difference between external $(M=33.29 \mathrm{mV}, S E=3.87 \mathrm{mV})$ and internal was approaching significance $(p=.056)$. For the tricep, pairwise comparisons showed no significant differences after Bonferroni correction, although the highest muscle activity was again observed in the internal condition $(M=5.20 \mathrm{mV}, S E=0.45 \mathrm{mV})$, with somatic $(M=4.86 \mathrm{mV}, S E=0.42 \mathrm{mV})$ and external $(M=4.85 \mathrm{mV}, S E=0.42 \mathrm{mV})$ being descriptively very similar).

For the bicep muscle, there was no effect of Focus Condition $(F(2,60)=0.33, p$ $=.719)$, no interaction effect, and no effect of Expertise $(F(1,30)=3.84, p=.060)$.

** Figure 8 about here **

\section{Relationships between variables}

As a final step, we explored relationships between physiological and physical variables with audio, in an attempt to gain insight into the mechanisms of sound-production. To this end, we correlated a) EMG activity of the three muscles and b) motion capture variables (bow velocity, acceleration, contact point, and scroll sway) with audio variables RMS, spectral centroid and roughness. We correlated only mean 
measurements, not standard deviations. For these purposes, the motion data was segmented to match the audio signal processing so that only motion during the bowing sections was represented. As some variables were not normally distributed we applied Spearman's correlations. For the EMG correlation block the alpha threshold for significance was adjusted to $p=.005$ ( 9 correlations) and for the motion capture block alpha was adjusted to $p=.004$ (12 correlations). We found no significant correlations between either EMG activity or motion features after alpha correction. This indicates that the audio measures were not directly related to either muscle activity or motion features, meaning that our results show different facets of FOA effects on soundproducing action.

\section{Discussion}

This study investigated effects of the psychological performance technique attentional focus on several stages of sound-producing action in both novice and experienced violinists, comparing effects of three attentional foci - internal (on arm movement), distal external (on sound), and somatic/proximal external (on bow-string resistance). We found significant effects of FOA for spectral centroid of violin sound, consistency of bow contact point, novices' violin scroll sway, and EMG activity of the deltoid and tricep muscles. These results exemplify how a change in psychological approach can impact motor control of sound production at several stages of the action-sound chain (See Figure 1), including an aspect of global motor behaviour (scroll sway). On the other hand, we found no effects of FOA for acoustic features RMS or roughness, bow acceleration, or bicep muscle activity. In support of our first hypothesis, we observed, on the whole, performance improvements under somatic (proximal external focus) compared to internal. However, we did not observe support for the distance effect, suggesting that the somatic (proximal external) focus on bow-string resistance was more 
beneficial to performance than the distal external focus on sound. Our second hypothesis was partly supported, with expertise significantly affecting several outcome variables, although we found that expertise influenced FOA effects only for the outcome measure violin sway, suggesting that FOA effects were largely independent from expertise.

\section{Acoustical outcomes}

We found a significant main effect of focus condition on the acoustic stage of sound production. Results showed that spectral centroid increased under a proximal external focus on tactile feedback, relative to an internal focus on arm movement. In contrast, we found no effects for RMS or roughness. As spectral centroid is widely agreed to be associated with perceived brightness of a sound (e.g. Trapasso, 2013), and violins with higher brightness have been judged as better quality by experts (Łukasik, 2005), we deemed a higher spectral centroid to indicate an improvement in tone quality. This finding thus supports our first hypothesis that performance outcomes would improve under external foci compared to internal. This is in line with the $\mathrm{CAH}$, previous research in sport (e.g. Neumann, 2019; Wulf, 2013; Wulf \& Lewthwaite, 2016) and music (Atkins, 2017; Duke et al., 2011; Mornell \& Wulf, 2019). However, this result does not support the distance effect that performance improves as FOA gets further from the body, as was observed by Duke, Cash, and Allen (2011) for a keyboard task, and Atkins (2017) for singing. In fact, we found no benefit of distal external focus on sound over an internal focus on arm movements in terms of producing a bright tone.

This result may be considered in light of Singh and Wulf's (2020) findings that a proximal external technique-based focus was more beneficial than distal external for those lacking in expertise (Singh \& Wulf, 2020). Regarding violin tone quality, perhaps focusing on sound is not a helpful external focus, even for experienced violinists, 
because it does not allow attention to the technical means of achieving the goal. The somatic focus may afford the benefit of drawing attention away from movement mechanisms (avoiding constrained action) while also bringing awareness to bow technique. This finding might also have implications for implicit learning in string playing, as the somatic focus might provide a method for violinists to discover how movements of the bow interact with the string without explicit instructions. Further research could test the efficacy of a somatic focus for learning in comparison to explicit instructions about arm or bow motion.

Unlike Singh and Wulf (2020), our findings for tone brightness applied to both experienced violinists and novices, but this may be a reflection of the complex nature of violin tone production, which is characterised by the careful balancing of several bowing parameters (Edgerton et al., 2014), and control of several degrees of freedom of motion (Konczak et al., 2009). The complexity of this motor skill may imply that even for experienced players, bowing movements are not fully automatised, meaning that an optimal external focus should bring attention to the technical means of sound production, rather than the sound itself. Additionally, our finding may have been different if we had a higher level of expertise in our sample. It would be interesting to explore if elite performers (e.g. professional soloists) might benefit from a sound focus where the less advanced players in the current study did not. Furthermore, violinists might benefit from a sound focus in other aspects of performance, such as timing, intonation, or expressing emotion in a melody. Another point to note about this finding is that spectral centroid in violin sound has been shown to be mainly influenced by bow force (Schoonderwaldt, 2009b), thus increased pressure of the bow into the string under the somatic focus may have underpinned this effect. Future studies should attempt to verify this by measuring changes in bow force due to attentional focus, which may be 
achieved through use of specially designed systems for tracking bowing parameters (Pardue, Harte, \& McPherson, 2015). This would be a useful avenue to explore for violin pedagogy, where teachers may wish to encourage increased bow pressure while avoiding explicit instructions, in line with discovery learning or implicit learning approaches (Bakker, 2018; Chambaron et al., 2009). In addition, as CAH theory is closely aligned with the explicit monitoring theory of choking under pressure (Beilock \& Carr, 2001), further research could explore effects of internal, external and somatic foci in music performance in a pressurised situation and consider results in the context of choking theories.

In contrast, we found no effects of FOA on RMS (i.e. loudness) or roughness (i.e. sensory dissonance) of sound produced. These contrasting findings reflect the multifaceted nature of even a simple string instrument sound production task in that there is a myriad of sound features which may or may not be affected. That we observed effects of attentional focus for spectral centroid and not the other features, indicates that bowing mechanics may be altered in a way that changes one aspect of tone but not others. This is consistent with mathematical understandings of bowing mechanics as a dynamic and complex system (e.g. Edgerton et al., 2014). Further research should explore the bowing features which might control the roughness of violin sound. Overall, our results indicate that a focus on tactile bowing sensations during violin playing can affect the acoustical output of the sound-producing action via an increase in the brightness of tone produced, but not through the RMS or roughness of the sound.

MIR feature selection is clearly an important process in the current paradigm, as the features chosen for analysis may define whether or not effects are observed. We based our feature selection on previous research, and aimed to select features that were reasonably well understood in terms of their perceptual attributes, but it is unlikely that 
FOA would affect all aspects of sound produced by a musician, and it is possible that other MIR features would have produced different results. As there is currently no standard acoustic measure to represent violin tone quality as a totality, it was a necessary limitation to focus on a select few features of tone. Further research exploring FOA in more expressive musical tasks with a fewer number of trials could utilise perceptual ratings. Nonetheless, the use of MIR features in the current study provides a reliable and quantifiable way of measuring changes in the mechanics of sound production, and is an important contribution to this field of research.

\section{Physical outcomes}

To assess effects of FOA on the physical stage of the sound-producing action, we examined technical bowing parameters of bow acceleration, bow contact point and violin sway. We found a main effect of focus condition on the consistency of bow contact point, such that bow contact point was less variable in the somatic condition relative to external. This finding indicates that a change in psychological approach may influence physical aspects of sound-producing motor control. In bowing technique, keeping the position of the bow on the string consistent (i.e. keeping the bow parallel to the bridge) is a well-established technical goal, in order to maintain a consistent tone (Fischer, 1997). Therefore, we considered lower standard deviation of bow contact point to indicate an improvement in bow control. Therefore, this result does not support our first hypothesis, as there was no difference between internal and either external condition, but rather points to a benefit of the somatic focus over the more distal sound focus. This is in line with our previous suggestion that the somatic focus encouraged awareness of bow technique, and is again in opposition to the distance effect (Singh \& Wulf, 2020), strengthening our proposition that a focus on tactile sensations from the instrument might be more helpful to violinists' tone production than a focus on the 
sound itself. This argument is supported by Stambaugh's $(2017 ; 2019)$ suggestion that awareness of tactile feedback is important for instrumental musicians and may be a contributing factor to FOA effects. This is also a pedagogically relevant finding, highlighting the usefulness of a somatic focus for improving measurable technical playing outcomes.

We found no effects of FOA on bow acceleration or mean bow contact point. A possible reason for the lack of any effect here may have been the very controlled nature of the task. For example, the instructions to play with a consistent tempo and bow amount may have left little room for variation in bow acceleration. The participants in the current study were all experienced musicians, and they appeared to adhere easily to these basic temporal and spatial parameters. Indeed it has been suggested that the CAH only applies to tasks which the performer finds difficult (Wulf, Töllner, \& Shea, 2013), and more variability in these parameters might have been observed in more complex, less controlled musical tasks, or with non-musician participants.

Results further demonstrated that attentional focus also significantly affected novices' freedom of body motion, as measured by micro changes in violin scroll sway. Novices' instrument sway significantly increased under somatic focus compared to internal, while experts were unaffected. The systematic changes in sway observed here, were a matter of millimetres in magnitude, suggesting changes in micro-motion rather than large swaying motions which could be disruptive to playing technique. As freedom of body motion is considered a positive pedagogical outcome (Roos, 2001), inhibiting overall body motion has been shown to negatively impact music performance (Rozé et al., 2020; Turner \& Kenny, 2011), and increases in micromotion while sitting have been associated with reductions in pain (Vergara \& Page, 2002), we interpreted increased instrument sway as representing subtle relaxations of posture and thus an improvement 
in freedom of body motion. This finding therefore supports our first hypothesis, with freer motion in proximal external relative to internal focus. Furthermore, the difference between somatic and external was approaching statistical significance, suggesting that the somatic focus also marginally improved sway compared to the sound focus. Experienced violinists exhibited significantly more sway than novices overall, meaning that novices' sway behaviour became closer to that of experienced players under the somatic focus, and supporting the interpretation of this effect as a performance improvement. This result sheds new light on how constrained action under an internal focus can lead to global changes in motor behaviour - i.e. changes to movement that are not specific to the part of the body focused on for the task. This is in line with previous findings that changes in FOA on a specific body part can affect motor behaviour in other parts of the body (Wulf \& Dufek, 2009). Indeed, it is argued that somatic training methods encourage reductions in stiffness through attention to subtle body sensations (Mattes, 2016), and our finding that the somatic focus increased sway may reflect similar mechanisms. Further research could build on this finding by exploring how changes in instrumental sway behaviour may affect perceptions of performance, or how FOA might affect larger gestural behaviour in expressive music performance.

\section{Physiological outcomes}

Our first hypothesis was also supported for the physiological stage of the sound production task. In line with previous research that an external focus promotes more efficient muscle use (e.g. Marchant \& Greig, 2017; Neumann \& Brown, 2013; Vance, Wulf, Töllner, McNevin, \& Mercer, 2004), we found significantly reduced muscle activity in the deltoid muscle (shoulder) under somatic focus compared to internal. This, to our knowledge, is novel evidence of this physiological effect in a music task. In somatic training methods, it is thought that attending to body sensations can reduce 
excess muscle tension, and it has further been suggested that this process is underpinned by the CAH (Mattes, 2016). Our findings support this, as focusing on tactile sensations through the violin bow caused decreased muscle activity in the right shoulder. Further research could explore how FOA affects muscle activity in specific muscles known to be problematic for certain instruments, and how this might be useful in preventing playing-related injuries.

However, we found no significant effects of FOA for the bicep muscle, and effects on the tricep muscle were not significant after Bonferroni correction. We suggest that the reason these muscles were not affected by attentional focus may have been because they followed an alternating activation pattern which allowed rest periods in which excess tension could dissipate. These rest periods may have negated any overactivation effects caused by the internal focus. Future research might further investigate how muscle activation patterns mediate increases in EMG activity as a result of constrained action.

\section{Expertise effects}

Our second hypothesis was that performance outcomes and effects of FOA would be different for experienced players and novices. This hypothesis was supported for several acoustic features, showing that, compared to novices, experienced violinists' sound was characterised as significantly less variable in spectral centroid, roughness and RMS, indicating greater control of sound consistency. Experienced players also played significantly quieter than novices and with less mean roughness, and their bow technique was characterised with higher variability of acceleration and a bow contact point further from the bridge. Also, experienced players' violin sway was greater than novices, indicating greater freedom of overall body motion. This distinct characterisation of experienced and novice players, even for a very simple task, is in 
line with evidence that violin bowing is a highly complex motor skill which may take years to master (Konczak et al., 2009). These findings can inform future studies that require parameters with which to measure quality of violin playing or to define violin expertise. In particular, lower mean roughness, lower standard deviation of MIR features, and higher violin sway may be useful features for characterising experienced players.

Our hypothesis that effects of FOA would be mediated by expertise was supported only for the violin sway measure. On all other measures no interaction of expertise and condition was observed. First, we suggest that the interaction effect of expertise and condition for instrument sway might indicate that the experienced violinists had learned to integrate sway behaviour with their playing in such a way that it would be unaffected by constrained action. Experienced players were likely very comfortable with the violin posture and found the bowing task relatively easy, meaning that even under an internal focus, their overall body motion remained free. On the other hand, novices were unfamiliar with the playing posture and may have therefore been more susceptible to constrained action effects on their swaying behaviour. Secondly, the lack of interaction effects in other measures supports previous findings in sport that the CAH may affect motor performance regardless of expertise (Wulf, 2013). However, as discussed earlier, our findings generally point to performance benefits under somatic/proximal external focus for both novices and experts rather than a more distal sound focus, and we believe that this is due to the complex nature of the sound production task which requires attention to technical means rather than to the end goal of sound. So, the lack of expertise effects observed here may be due to the complex nature of violin tone production, and indeed, a sample of violinists with a wider range of expertise (i.e. elite solo performers) may yield different results. A final point to note on 
expertise is that it has been shown that training in certain musical skills, such as focussing on various instruments in an orchestra simultaneously, affects attentional capacities (Wöllner \& Halpern, 2016). In this study, conductors had better divided attention skills compared to pianists, while more experienced musicians outperformed less experienced ones. In the current sample it is unknown if the experienced violinist group had better attentional capacities than the other group, and therefore might have been better at following the focus instructions. Future research using this paradigm could take individual differences in attentional capacity into consideration.

\section{Relationships between variables}

As a final exploratory measure, we investigated the relationships between physiological muscle activity and motion variables with MIR features. We observed no significant correlations between motion or EMG measures and audio features, showing that the measures taken at different stages of the sound-producing action chain represent different aspects of the action. Although it may seem surprising that bow motion features did not correlate with MIR outcomes, these relationships may require measurement of other variables such as bow force, and flatness of bow hair, in order to understand them fully. While previous research has suggested that bow contact point is related to loudness (i.e. RMS, Edgerton et al., 2014), the lack of this relationship here might be explained by a lack of variation in these parameters due to the strict nature of the task. Gaining a full picture of how motion and physiological parameters contribute to violin sound production would be a useful topic for further research.

\section{Limitations}

Several limitations of the current study should be considered. Firstly, the task used was a reductive technical exercise not representative of the full scope of what music 
performance encompasses. Nonetheless, the exercise was a realistic one, important to the early stages of learning to play the violin, and has provided key findings which can inform further study of more expressive, complex musical tasks. It should also be noted that the current study examined only performance effects of FOA, not learning effects. Exploring FOA effects on the learning of violin bowing skills would be a suitable topic for further research. Finally, the current study took place in a laboratory, where participants were required to wear various body sensors, which is undoubtedly an unusual music performing environment, and results may differ in a more naturalistic setting. Future research could thus build on the current findings by aiming to replicate them outside of the laboratory, with more complex, expressive musical tasks.

\section{Conclusions}

This study provides novel evidence that the psychological performance approach of attentional focus can affect physiological, physical and acoustical aspects of motor control during a violin sound production task, in both experts and novices. Results also showed that attentional focus affected a more global aspect of motor control, namely freedom of body motion measured through instrument sway, for novices but not experts. Our findings provide support for the $\mathrm{CAH}$ in violin sound production (a continuous instrumental sound production task), in line with previous FOA research in sport. We found no evidence for the distance effect, thus we propose that the complex motor skills of violin tone production benefit from a proximal external focus which allows attention to the technical means of sound production (Singh \& Wulf, 2020). Furthermore, the performance benefits we found of focusing on tactile sensation, support putative mechanisms of somatic training methods for improving performance by encouraging awareness of body sensations and movement habits (Mattes, 2016). We additionally found that attentional effects were modulated by expertise only for the 
freedom of body motion measure, indicating that aspects of attentional focus effects on sound production may occur regardless of violin playing expertise. Future research on this topic should investigate effects of attentional focus on expressive musical outcomes, and in situations of psychological pressure, as well as possible connections of attentional focus effects on muscle activity with playing related injuries, and the use of a tactile focus as an implicit learning strategy.

\section{Funding details}

This work was supported by a Consolidator Grant to the third author from the European Research Council under Grant 725319. The funders had no role in study design, data collection and analysis, decision to publish, or preparation of the manuscript.

\section{References}

Alishah, E. R., Ates, O., \& Ahmadi, M. (2017). The effects of attentional focus on the performance of volleyball jump serve in elite players. Physical Education and Sport Science, 3(12), 48-58. https://doi.org/10.5281/zenodo.1066314

Alluri, V., \& Toiviainen, P. (2009). Exploring perceptual and acoustical correlates of polyphonic timbre. Music Perception, 27(3), 223-242. https://doi.org/https://doi.org/10.1525/mp.2010.27.3.223

Atkins, R. (2017). Effects of focus of attention on tone production in trained singers. Journal of Research in Music Education, 64(4), 421-434. https://doi.org/10.1177/0022429416673842

Bakker, A. (2018). Discovery learning: zombie, phoenix, or elephant? Instructional Science, 46(1), 169-183. https://doi.org/10.1007/s11251-018-9450-8

Batson, G. (2007). Revisiting overuse injuries in dance in view of motor learning and somatic models of distributed practice. Journal of Dance Medicine \& Science, 11(3), 70-75. https://doi.org/https://doi.org/10.1037/0096-3445.130.4.701

Beilock, S. L., \& Carr, T. H. (2001). On the fragility of skilled performance: what governs choking under pressure? Journal of Experimental Psychology: General, 
130(4), 701-725.

Bell, J. J., \& Hardy, J. (2009). Effects of attentional focus on skilled performance in golf. Journal of Applied Sport Psychology, 21(2), 163-177. https://doi.org/10.1080/10413200902795323

Buma, L. A., Bakker, F. C., \& Oudejans, R. R. D. (2015). Exploring the thoughts and focus of attention of elite musicians under pressure. Psychology of Music, 43(4), 459-472. https://doi.org/10.1177/0305735613517285

Burger, B., \& Toiviainen, P. (2013). MoCap Toolbox - A Matlab toolbox for computational analysis of movement data. In R. Bresin (Ed.), Proceedings of the sound and music computing conference 2013 (pp. 172-178). Berlin: Logos Verlag. https://doi.org/http://urn.fi/URN:NBN:fi:jyu-201401211091

Burkholder, K. R., \& Brandfonbrener, A. G. (2004). Performance-related injuries among student musicians at a specialty clinic. Medical Problems of Performing Artists, 19(3), 116-123.

Chambaron, S., Berberian, B., Delbecque, L., Ginhac, D., \& Cleeremans, A. (2009). Implicit motor learning in discrete and continuous tasks: Toward a possible account of discrepant results. In L. T. Pelligrino (Ed.), Handbook of Motor Skills:Development, Impairment and Therapy (pp. 139-155). Nova Science Publishers.

Chudy, M. (2016). Discriminating music performers by timbre: On the relation between instrumental gesture, tone quality and perception in classical cello performance (Doctoral Thesis). Retrieved from: http://qmro.qmul.ac.uk/xmlui/handle/123456789/18378.

Cotik, T. (2019). Concepts of the Alexander Technique and practical ideas for musicians. American String Teacher, 69(2), 33-36. https://doi.org/10.1177/0003131319835543

Dahl, S., \& Friberg, A. (2007). Visual perception of expressiveness in musicians' body movements. Music Perception, 24(5), 433-454. https://doi.org/10.1525/mp.2007.24.5.433

Davies, J. (2020). Alexander Technique classes improve pain and performance factors in tertiary music students. Journal of Bodywork and Movement Therapies, 24(1), 
1-7. https://doi.org/10.1016/j.jbmt.2019.04.006

Desmet, F., Nijs, L., Demey, M., Lesaffre, M., Martens, J.-P., \& Leman, M. (2012). Assessing a clarinet player's performer gestures in relation to locally intended musical targets. Journal of New Music Research, 41(1), 31-48. https://doi.org/10.1080/09298215.2011.649769

Deutsch, L. M. (2011). Motion study of violin bowing technique: a study comparing the motor patterns of professional and student violinists (Doctoral Dissertation). Available from ProQuest Dissertations Publishing (UMI Number: 3497390)

Dries, K., Vincken, W., Loeckx, J., Schuermans, D., \& Dirckx, J. (2017). Effects of a respiratory muscle training program on respiratory function and musical parameters in saxophone players. Journal of New Music Research, 46(4), 381-393. https://doi.org/10.1080/09298215.2017.1358751

Duke, R. A., Cash, C. D., \& Allen, S. E. (2011). Focus of attention affects performance of motor skills in music. Journal of Research in Music Education, 59(1), 44-55. https://doi.org/10.1177/0022429410396093

Edgerton, M. E., Hashim, N., \& Auhagen, W. (2014). A case study of scaling multiple parameters by the violin. Musicae Scientiae, 18(4), 473-496. https://doi.org/10.1177/1029864914550666

Eerola, T., Ferrer, R., \& Alluri, V. (2012). Timbre and affect dimensions: Evidence from affect and similarity ratings and acoustic correlates of isolated instrument sounds. Music Perception, 30(1), 49-70. https://doi.org/https://doi.org/10.1525/mp.2012.30.1.49

Fischer, S. (1997). Basics: 300 excercises and practice routines for the violin. Leipzig, Germany: Edition Peters.

Giraldo, S., Ramirez, R., Waddell, G., \& Williamon, A. (2017). A Real-time feedback learning tool to visualize sound quality in violin performances. In R. Ramirez, D. Conklin, \& J. M. Iñesta (Eds.), 10th International Workshop on Machine Learning and Music (pp. 19-24). Barcelona, Spain.

Giraldo, S., Waddell, G., Nou, I., Ortega, A., Mayor, O., Perez, A., Williamon, A., \& Ramirez, R. (2019). Automatic assessment of tone quality in violin music performance. Frontiers in Psychology, 10, 1-12. 
https://doi.org/10.3389/fpsyg.2019.00334

Hove, M. J., Vuust, P., \& Stupacher, J. (2019). Increased levels of bass in popular music recordings 1955-2016 and their relation to loudness. The Journal of the Acoustical Society of America, 145(4), 2247-2253. https://doi.org/10.1121/1.5097587

Ives, J. C. (2003). Comments on "the feldenkrais method $\AA$ : A dynamic approach to changing motor behavior." Research Quarterly for Exercise and Sport, 74(2), 116123. https://doi.org/10.1080/02701367.2003.10609072

Jensenius, A. R. (2007). Action-sound: Developing methods and tools to study musicrelated body movement (Doctoral Thesis). Retrieved from http://urn.nb.no/URN:NBN:no-18922

Kokotsaki, D., \& Davidson, J. W. (2003). Investigating musical performance anxiety among music college singing students: A quantitative analysis. Music Education Research, 5(1), 45-59. https://doi.org/10.1080/14613800307103

Konczak, J., Vander Velden, H., \& Jaeger, L. (2009). Learning to play the violin: motor control by freezing, not freeing degrees of freedom. Journal of Motor Behavior, 41(3), 243-252. https://doi.org/10.3200/JMBR.41.3.243-252

Lartillot, O., \& Toiviainen, P. (2007). A Matlab toolbox for musical feature extraction from audio. In International Conference on Digital Audio Effects. Bordeaux.

Lee, C. (2018). Musicians as movers: Applying the Feldenkrais method to music education. Music Educators Journal, 104(4), 15-19. https://doi.org/10.1177/0027432118766401

Łukasik, E. (2005). Towards timbre-driven semantic retrieval of violins. In H. Kwaskicka \& M. Paprzycki (Eds.), 5th International Conference on Intelligent Systems Design and Applications (pp. 55-60). Warsaw, Poland: IEEE. https://doi.org/10.1109/ISDA.2005.94

Marchant, D. C., \& Greig, M. (2017). Attentional focusing instructions influence quadriceps activity characteristics but not force production during isokinetic knee extensions. Human Movement Science, 52, 67-73. https://doi.org/10.1016/j.humov.2017.01.007

Mattes, J. (2016). Attentional focus in motor learning, the feldenkrais method, and mindful movement. Perceptual and Motor Skills, 123(1), 258-276. 
https://doi.org/10.1177/0031512516661275

McKay, B., \& Wulf, G. (2012). A distal external focus enhances novice dart throwing performance. International Journal of Sport and Exercise Psychology, 10(2), 149156. https://doi.org/10.1080/1612197X.2012.682356

McNevin, N. H., Shea, C. H., \& Wulf, G. (2002). Increasing the distance of an external focus of attention enhances learning. Psychological Research, 67(1), 22-29. https://doi.org/10.1007/s00426-002-0093-6

Medoff, L. E. (1999). The importance of movement education in the training of young violinists. Medical Problems of Performing Artists, 14(4), 210-219.

Mornell, A., \& Wulf, G. (2019). Adopting an external focus of attention enhances musical performance. Journal of Research in Music Education, 66(4), 375-391. https://doi.org/10.1177/0022429418801573

Nagyvary, J. (2013). A Comparative Study of Power Spectra and Vowels in Guarneri Violins and Operatic Singing. Bulletin of the American Physical Societymerican Physical Society, 1(3), 1-30.

Neumann, D., \& Brown, J. (2013). The effect of attentional focus strategy on physiological and motor performance during a sit-up exercise. Journal of Psychophysiology, 27(1), 7-15. https://doi.org/10.1027/0269-8803/a000081

Neumann, D. L. (2019). A systematic review of attentional focus strategies in weightlifting. Frontiers in Sports and Active Living, 1(7), 1-7. https://doi.org/10.3389/fspor.2019.00007

Pardue, L. S., Harte, C., \& McPherson, A. P. (2015). A low-cost real-time tracking system for violin. Journal of New Music Research, 44(4), 305-323. https://doi.org/10.1080/09298215.2015.1087575

Perez-Carrillo, A. (2016). Statistical models for the indirect acquisition of violin bowing controls from audio analysis. Proceedings of Meetings on Acoustics, 29(1), 1-9. https://doi.org/10.1121/2.0000402

Perez-Carrillo, A. (2019). Violin timbre navigator: Real-time visual feedback of violin bowing based on audio analysis and machine learning. In I. Kompatsiaris, B. Huet, V. Mezaris, C. Gurrin, W. H. Cheng, \& S. Vrochidis (Eds.), 25th International Conference on Multimedia Modeling, Proceedings, Part II (pp. 182-193). 
Thessaloniki, Greece: Springer. https://doi.org/10.1007/978-3-030-05716-9_15

Porter, J. M., Anton, P. M., \& Wu, W. F. W. (2012). Increasing the Distance of an External Focus of Attention Enhances Standing Long Jump Performance. Journal of Strength and Conditioning Research, 26(9), 2389-2393. https://doi.org/10.1519/JSC.0b013e31823f275c

Riley, M. A., Stoffregen, T. A., Grocki, M. J., \& Turvey, M. T. (1999). Postural stabilization for the control of touching. Human Movement Science, 18(6), 795817. https://doi.org/10.1016/S0167-9457(99)00041-X

Roos, J. W. (2001). Violin playing: Teaching freedom of movement (Master's Dissertation). Retrieved from https://repository.up.ac.za/bitstream/handle/2263/30005/dissertation.pdf;sequence= 1

Rozé, J., Aramaki, M., Kronland-Martinet, R., \& Ystad, S. (2020). Cellists' sound quality is shaped by their primary postural behavior. Scientific Reports, 10(1), 117. https://doi.org/10.1038/s41598-020-70705-8

Schoonderwaldt, E. (2009a). Mechanics and acoustics of violin bowing: Freedom, constraints and control in performance (Doctoral Thesis). Retrieved from https://www.diva-portal.org/smash/record.jsf?pid=diva2\%3A133385\&dswid=8078

Schoonderwaldt, E. (2009b). The violinist's sound palette: spectral centroid, pitch flattening and anomolous low frequencies. Acta Acustica, 95(5), 901-914. https://doi.org/https://doi.org/10.3813/AAA.918221

Shea, C. H., Wulf, G., Whitacre, C. A., \& Park, J. H. (2001). Surfing the implicit wave. Quarterly Journal of Experimental Psychology Section A: Human Experimental Psychology, 54(3), 841-862. https://doi.org/10.1080/713755993

Shusterman, R. (2009). Body consciousness and performance: Somaesthetics east and west. Journal of Aesthetics and Art Criticism, 67(2), 133-145. https://doi.org/10.1111/j.1540-6245.2009.01343.x

Singh, H., \& Wulf, G. (2020). The distance effect and level of expertise: Is the optimal external focus different for low-skilled and high-skilled performers? Human Movement Science, 73, 1-6. https://doi.org/10.1016/j.humov.2020.102663

Slade, T., Comeau, G., \& Russell, D. (2020). Measurable changes in piano performance 
of scales and arpeggios following a Body Mapping workshop. Journal of New Music Research, 49(4), 362-372. https://doi.org/10.1080/09298215.2020.1784958

Stambaugh, L. A. (2017). Effects of internal and external focus of attention on woodwind performance. Psychomusicology: Music, Mind, and Brain, 27(1), 4553. https://doi.org/10.1037/pmu0000170

Stambaugh, L. A. (2019). Effects of focus of attention on performance by second-year band students. Journal of Research in Music Education, 67(2), 1-14. https://doi.org/10.1177/0022429419835841

Trapasso, L. (2013). Feature-based Analysis of the Violin Tone Quality (Master's Thesis). Retrieved from http://hdl.handle.net/10589/89882

Turner, G., \& Kenny, D. T. (2011). Voluntary restraint of body movement potentially reduces overall SPL without reducing SPL range in western contemporary popular singing. Journal of New Music Research, 40(4), 367-378. https://doi.org/10.1080/09298215.2011.594513

Vance, J., Wulf, G., Töllner, T., McNevin, N., \& Mercer, J. (2004). EMG activity as a function of the performer's focus of attention. Journal of Motor Behavior, 36(4), 450-459. https://doi.org/10.3200/JMBR.36.4.450-459

Vergara, M., \& Page, Á. (2002). Relationship between comfort and back posture and mobility in sitting-posture. Applied Ergonomics, 33(1), 1-8. https://doi.org/10.1016/S0003-6870(01)00056-4

Wöllner, C., \& Halpern, A. R. (2016). Attentional flexibility and memory capacity in conductors and pianists. Attention, Perception, and Psychophysics, 78(1), 198208. https://doi.org/10.3758/s13414-015-0989-z

Wulf, G. (2013). Attentional focus and motor learning: A review of 15 years. International Review of Sport and Exercise Psychology, 6(1), 77-104. https://doi.org/10.1080/1750984X.2012.723728

Wulf, G., \& Dufek, J. S. (2009). Increased jump height with an external focus due to enhanced lower extremity joint kinetics. Journal of Motor Behavior, 41(5), 401409. https://doi.org/10.1080/00222890903228421

Wulf, G., \& Lewthwaite, R. (2016). Optimizing performance through intrinsic motivation and attention for learning: The OPTIMAL theory of motor learning. 
Psychonomic Bulletin and Review, 23(5), 1382-1414.

https://doi.org/10.3758/s13423-015-0999-9

Wulf, G., McNevin, N., \& Shea, C. H. (2001). The automaticity of complex motor skill learning as a function of attentional focus. Quarterly Journal of Experimental Psychology Section A: Human Experimental Psychology, 54(4), 1143-1154. https://doi.org/10.1080/713756012

Wulf, G., Töllner, T., \& Shea, C. H. (2013). Attentional focus effects as a function of task difficulty. Research Quarterly for Exercise and Sport, 78(3), 257-264. https://doi.org/10.1080/02701367.2007.10599423 
Table 1. Results of mixed ANOVAs for control measures length of task, bow cumulative distance travelled, bow velocity

$$
\text { Degrees of freedom } \quad \mathrm{F} \text { value } \mathrm{P} \text { value } \quad \eta^{2} p
$$

\begin{tabular}{llccc}
\hline Length of task & $1.32,39.62$ & 0.53 & .521 & .017 \\
Bow velocity & $1.60,47.86$ & 1.04 & .348 & .033 \\
\hline
\end{tabular}

* denotes a statistically significant effect. Mauchley's test of sphericity was significant $(p<.001)$ for both measures, so the Greenhouse-Geisser corrections are reported 
Table 2. List of MIR features used to represent changes in tone quality

\begin{tabular}{|c|c|c|c|}
\hline Feature & Description & Explanation & References \\
\hline Spectral & Measure of the & Associated with & (Edgerton et al., 2014; \\
\hline \multirow[t]{3}{*}{ centroid } & gravitational centre of & perceived brightness, & Schoonderwaldt, \\
\hline & the spectral & and bow pressure in & 2009b) \\
\hline & distribution of a sound & violin playing & \\
\hline \multirow[t]{5}{*}{ Roughness } & Measure of sensory & An estimate of sensory & (Eerola et al., 2012) \\
\hline & dissonance caused by & dissonance & \\
\hline & the "beating" of two & & \\
\hline & sinusoids in the same & & \\
\hline & critical band & & \\
\hline \multirow[t]{3}{*}{ RMS } & Root mean square of & A rough measure of the & (Hove et al., 2019) \\
\hline & amplitude, measure of & loudness of a sound & \\
\hline & the energy of the signal & & \\
\hline
\end{tabular}


Table 3. Expertise Results of mixed ANOVAs for spectral centroid, spectral flux, roughness, and fullness.

\begin{tabular}{llllll}
\hline & & & & Experienced & Novice \\
\hline Measure & F value & P value & $\eta^{2} p$ & $M(S E)$ & $M(S E)$ \\
\hline M spectral centroid & $0.17(1,25)$ & .684 & .007 & $1382.12(42.55)$ & $1356.84(44.12)$ \\
SD spectral centroid & $7.12(1,26)$ & $\mathbf{. 0 1 3} *$ & .22 & $111.81(13.94)$ & $164.41(13.94)$ \\
M RMS & $5.14(1,27)$ & $\mathbf{. 0 3 2} *$ & .16 & $0.08(0.01)$ & $0.12(0.01)$ \\
$S D$ RMS & $6.57(1,29)$ & $\mathbf{. 0 1 6 *}$ & .19 & $0.02(0.01)$ & $0.04(0.004)$ \\
M Roughness & $5.97(1,25)$ & $\mathbf{. 0 2 2 *}$ & .44 & $0.04(0.01)$ & $0.09(0.01)$ \\
$S D$ Roughenss & $7.02(1,25)$ & $\mathbf{. 0 1 4} *$ & .22 & $0.03(0.01)$ & $0.08(0.02)$ \\
\hline
\end{tabular}

* denotes a statistically significant effect. 


\section{Figures}

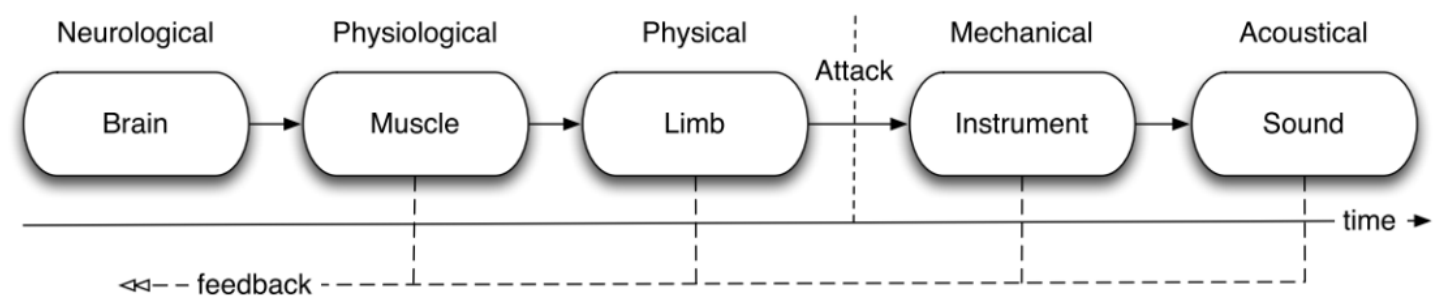

Figure 1.

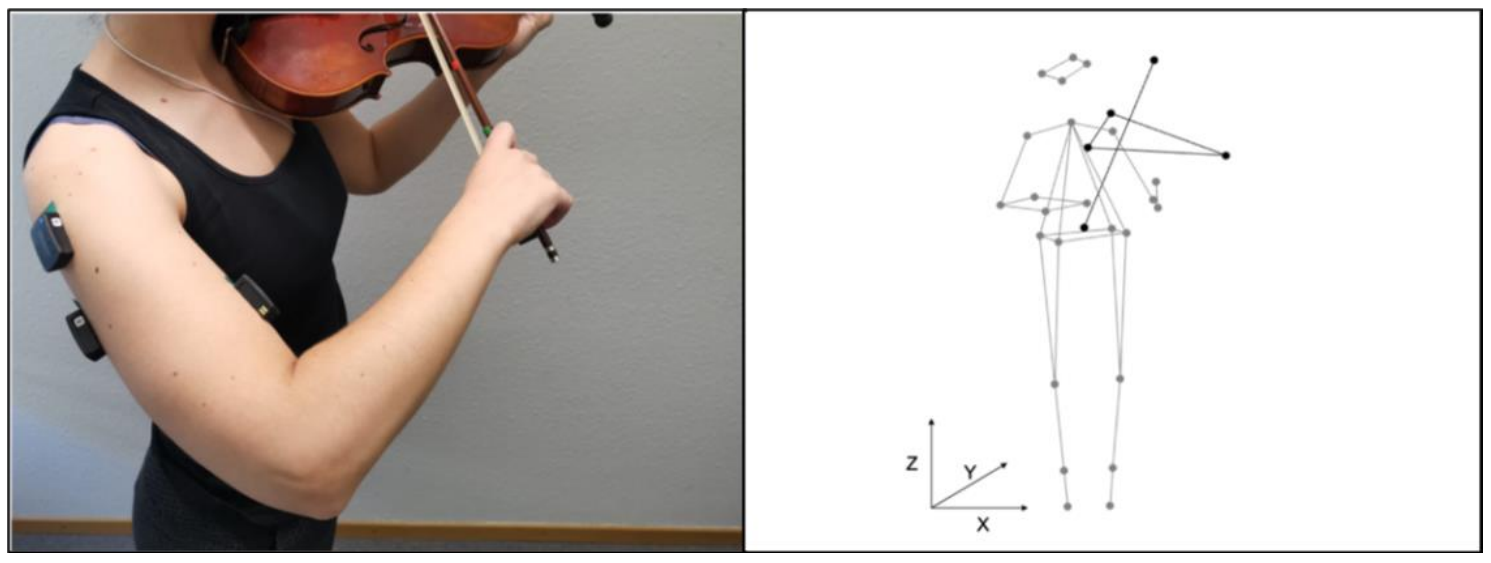

Figure 2.

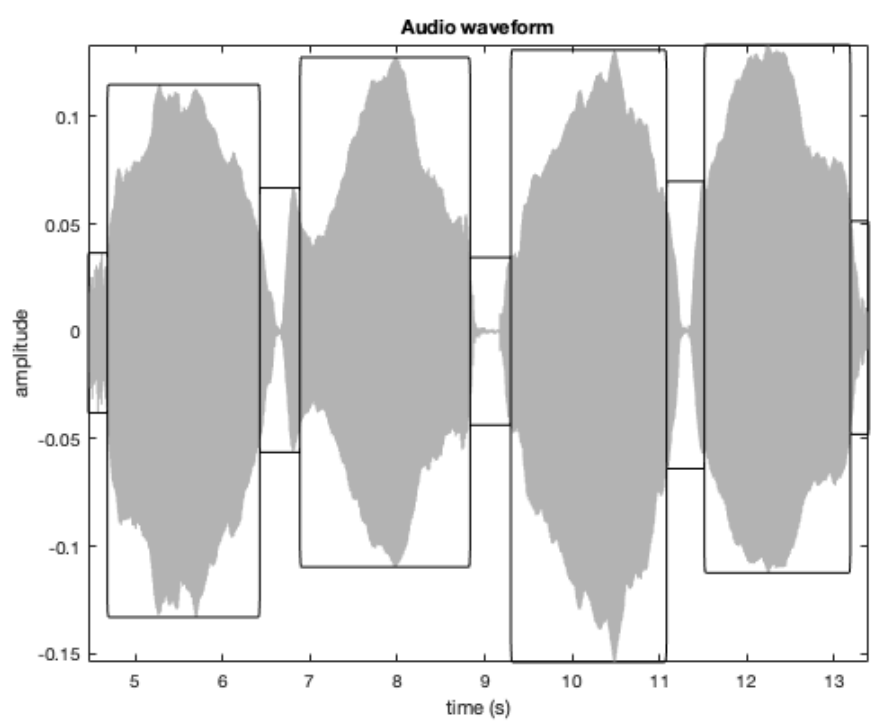

Figure 3. 


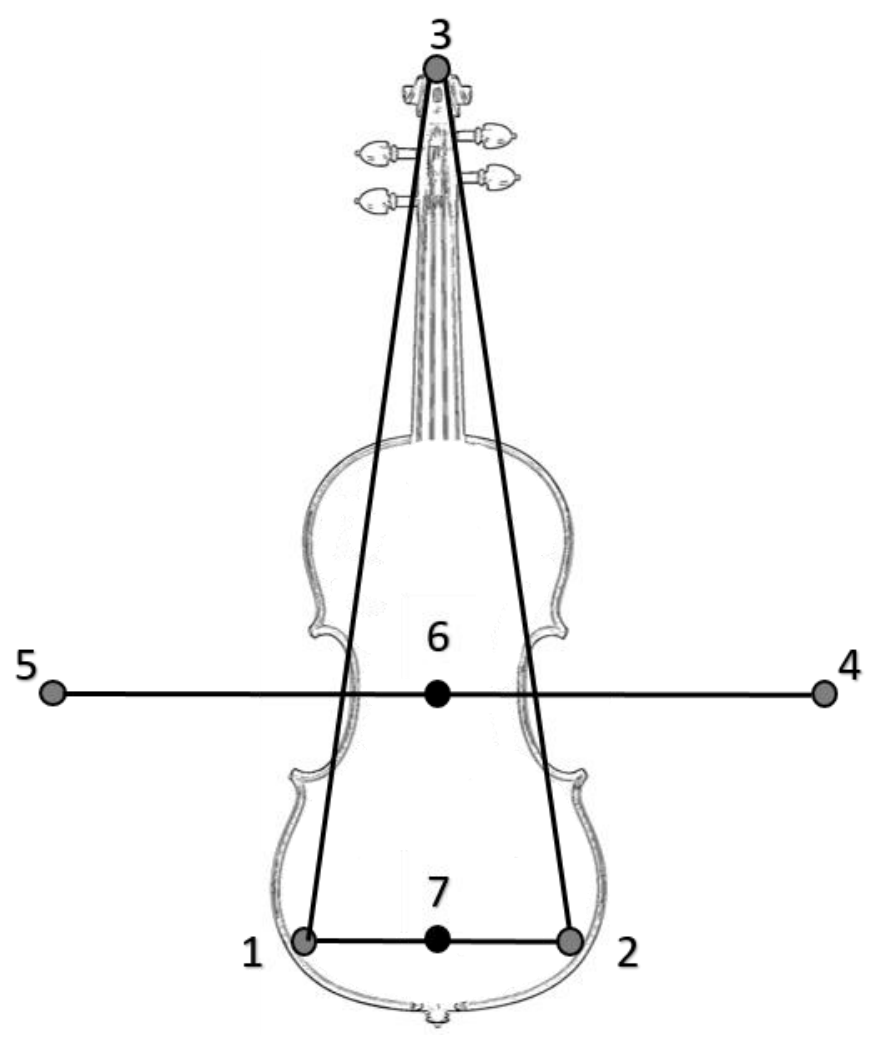

Figure 4.

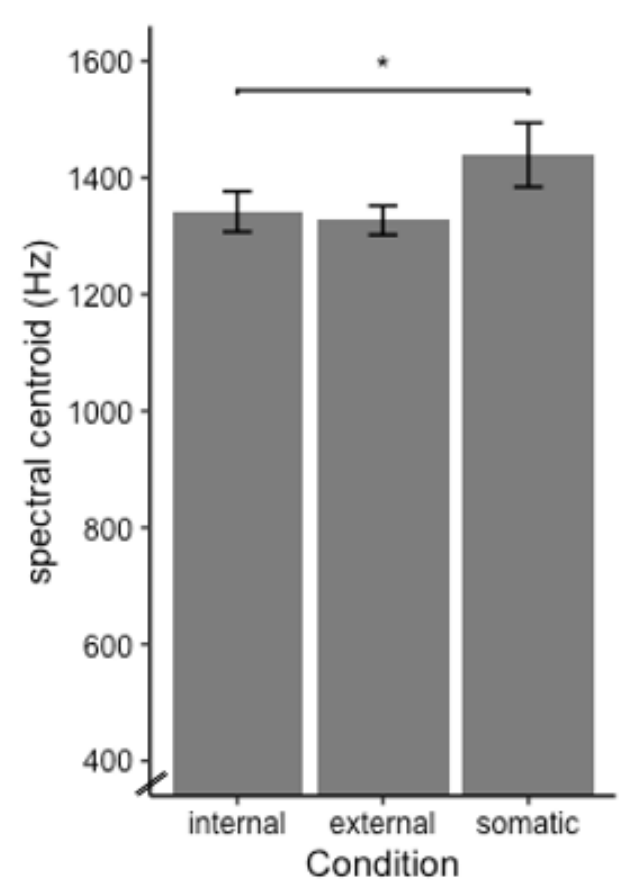

Figure 5. 


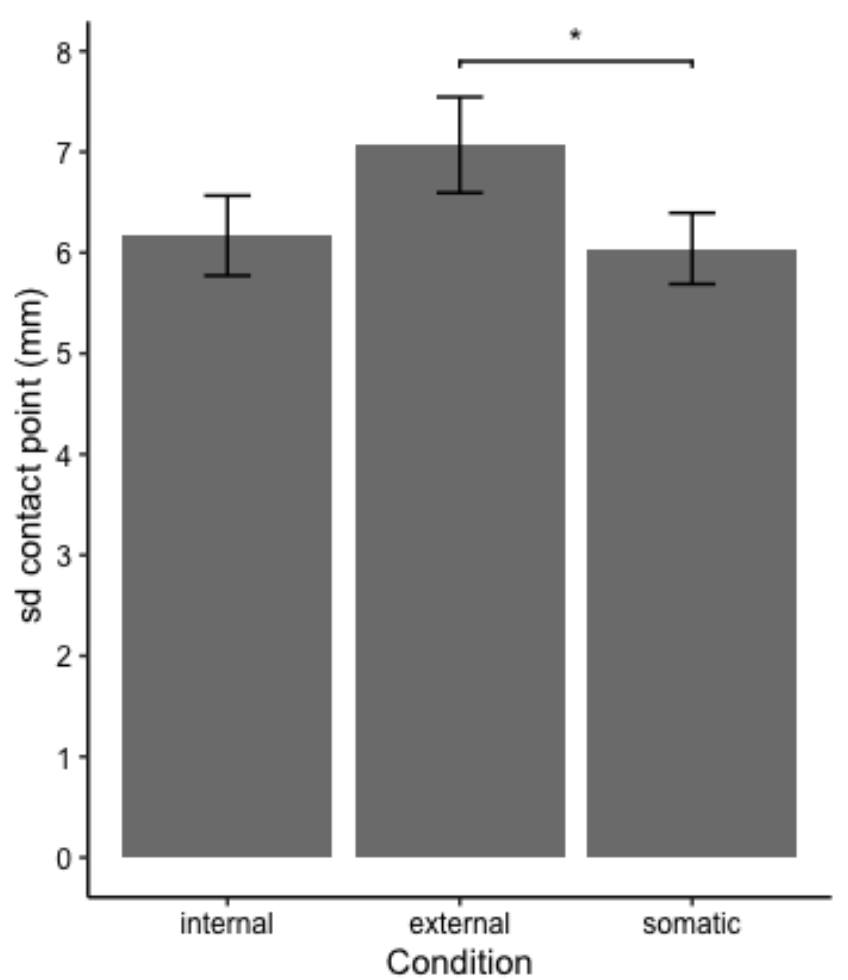

Figure 6

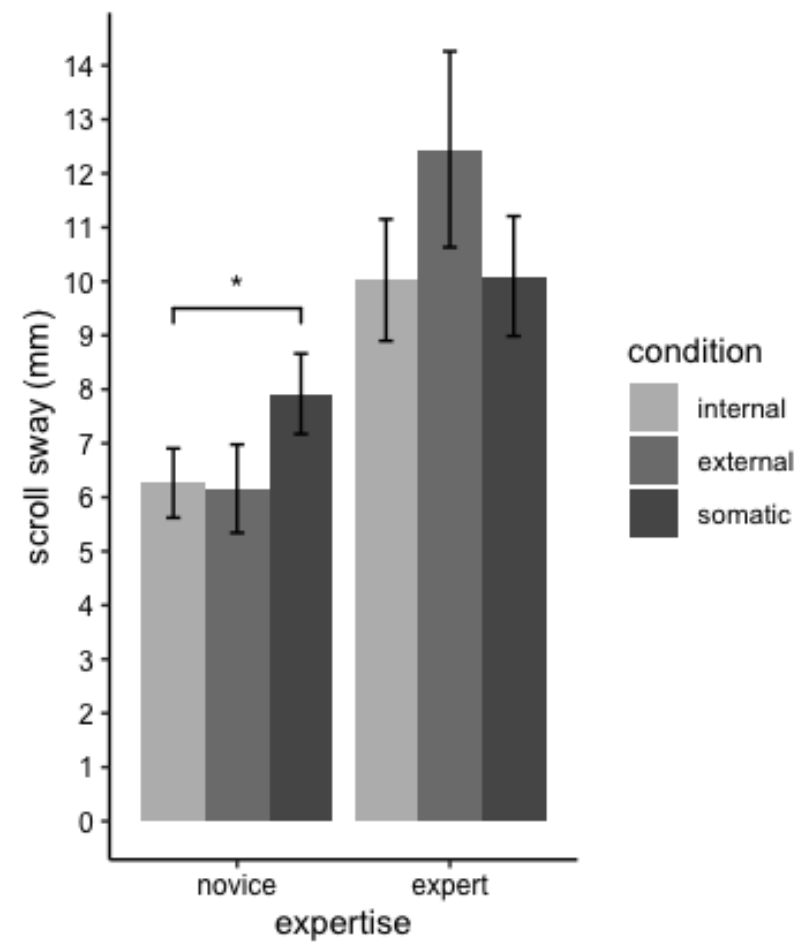

Figure 7 

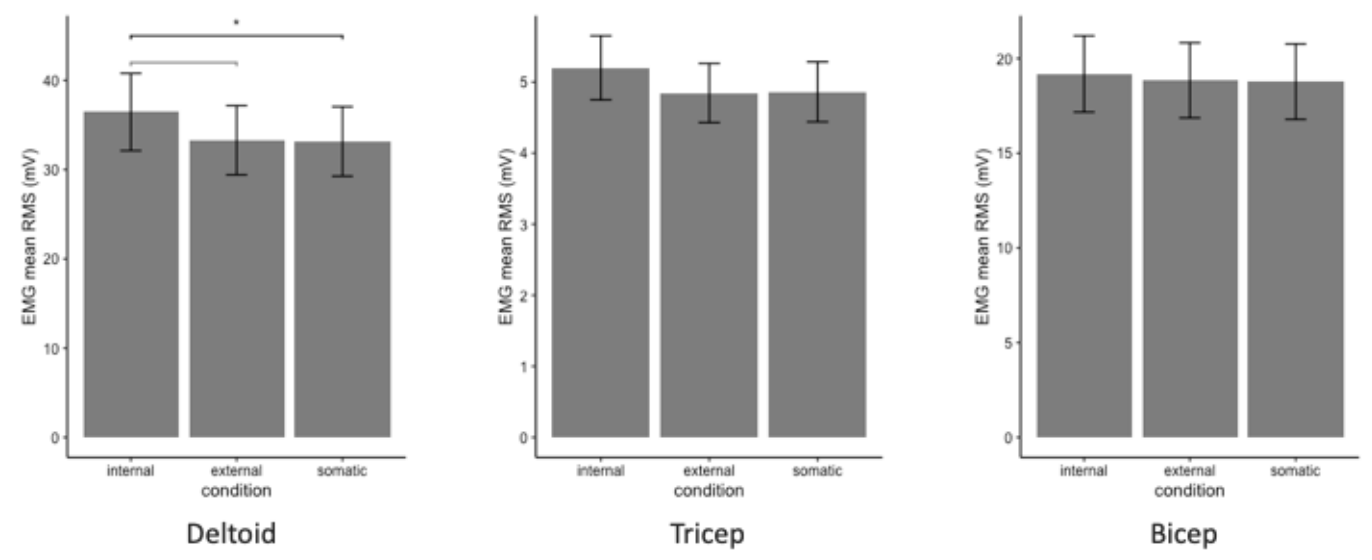

Figure 8.

\section{Figure Captions}

Figure 1. Action-sound chain as depicted by Jensenius (2007)

Figure 2. The left panel shows placement of EMG sensors on the bicep, tricep and deltoid muscles. The right panel shows motion capture markers placed on the participant's body, violin, and bow, with $\mathrm{X}, \mathrm{Y}$ and $\mathrm{Z}$ axes depicted in the bottom left corner.

Figure 3. Segmentation of audio waveform into "bow" (larger sections) and "bow change" (smaller sections) segments.

Figure 4. Motion capture violin markers. The grey markers indicate the physical markers placed on the violin and bow, and the black markers indicate the "virtual" markers used for bow analysis. The markers are: 1) left base, 2) right base, 3) scroll, 4) bow heel, 5) bow point, 6) bow mid, 7) mid base.

Figure 5 Effects of Focus Condition on mean spectral centroid. * denotes a statistically significant difference, $p<.05$. Error bars indicate standard error of the mean.

Figure 6. Effect of FOA on standard deviation of bow contact point. * denotes a statistically significant difference, $p<.05$. Error bars indicate standard error of the mean. 
Figure 7. Significant effect of focus condition on scroll sway for novices. $*$ denotes a statistically significant difference, $p<.05$. Error bars indicate standard error of the mean.

Figure 8. Focus Condition effects on EMG activity in deltoid, tricep, and bicep. * denotes a statistically significant difference, $p<.05$. Grey significance line denotes a difference approaching statistical significance. Error bars indicate standard error of the mean. 